\title{
Perfil del Paciente Diabético Tipo 2 de 55 y Más Años, de una Clínica Periférica de la Caja Costarricense de Seguro Social
}

\author{
Adriana Laclé Murray*
}

\begin{abstract}
La Diabetes mellitus es una enfermedad crónica cuya prevalencia aumentará con el envejecimiento de la población costarricense. El pronóstico y la calidad de vida de estos pacientes dependen de sus complicaciones crónicas que comienzan a presentarse después de los 55 años. Es necesario conocer el perfil de estos pacientes para determinar la mejor forma de brindarles la atención en salud que responda a sus necesidades. En 1986 se creó en la Clínica Marcial Fallas de Desamparados una modalidad de atención denominada "Programa de Enfermedades Crónicas". Además de la consulta médica especializada, se cuenta con una preconsulta, una clínica del pie diabético y un Club de Diabéticos. Se describe en este trabajo el perfil sociodemográfico, patológico y control metabólico del paciente diabético no insulino dependiente de 55 años y más, que asiste a PROENCRO. En 1995 se tenía registrado un total de 603 pacientes, de los cuales 432 (71.6\%) eran DM tipo 2, con 55 años o más. Esta población se caracterizó por ser en la mayoría mujeres ( $71 \%$ ), de baja escolaridad, de bajo nivel económico (28\% asegurados por el estado), con alta prevalencia de patologías crónicas como hipertensión arterial (61.9\%), obesidad (78.5\%, incluyendo sobrepeso) y dislipidemia $(41.5 \%)$. Las complicaciones secundarias fueron importantes: retinopatía en aproximadamente un $25 \%$ y nefropatía en un $8 \%$. El riesgo de sufrir patología grave (úlceras, mal perforante o amputación) en sus miembros inferiores fue de casi el 45\% (Pie amarillo en PATONA).

Su perfil metabólico fue insatisfactorio: el $72 \%$ de los pacientes tuvieron un promedio anual de glicemias mayor a 140 $\mathrm{mg} \%$, el $75 \%$ colesterol $>200 \mathrm{mg} \%$ y $67.3 \%$ un LDL-colesterol $>130 \mathrm{mg} \%$.

Ante este panorama debemos buscar nuevas estrategias de atención integral en salud, ya que esta va más allá de solo la atención médica. Debe enfatizarse la promoción y la prevención en salud.
\end{abstract}

Descriptores: adulto mayor, diabetes mellitus, control metabólico, antropometría, complicaciones secundarias.

En Costa Rica se hả logrado reducir notablemente la morbimortalidad provocada por las enfermedades infecto-contagiosas y parasitarias, debido a un mejoramiento en las condiciones sanitarias y la atención primaria y secundaria, además del cambio en las condiciones sociales y económicas de la población costarricense.

Abreviaturas: DM: Diabetes mellitus, PROENCRO: Programa de Enfermedades Crónicas, ALAD: Asociación Latinoamericana de Diabetes, C.C.S.S.: Caja Costarricense de Seguro Social, HTA: Hipertensión Arterial, H: Hombres, M: Mujeres, H.O. Hipoglicemiantes orales, IMC: Indice de Masa Corporal.

* Instituto de Investigaciones en Salud, Universidad de Costa Rica.

Correspondencia: Adriana Laclé, INISA, Universidad de Costa Rica.

46 AMC, junio 1999, vol 41 (2)
El perfil epidemiológico ha comenzado a evidenciar un predominio de enfermedades propias de un país desarrollado, entre ellas encontramos la Diabetes mellitus (DM). Esta enfermedad representa actualmente cerca del $2,8 \%$ del total de consultas médicas ambulatorias del país, ${ }^{1}$ una tasa de 13.4/ 10.000 habitantes en egresos hospitalarios, ${ }^{2}$ y en cuanto a mortalidad se ha mantenido entre las primeras diez causas de muerte general, con una tasa de 12.8 por 100.000 en $1996,{ }^{3}$ tasa de mortalidad que tiene un subregistro no cuantificado, ya que este diagnóstico usualmente no se especifica en el registro de defunción como causa asociada a la principal, como sucede en muchos casos con la cardiopatía isquémica, por lo cual se deduce que su importancia es mayor que la consignada.

La DM es una enfermedad crónica cuya incidencia y prevalencia aumentarán con el envejecimiento de la población costarricense en las próximas décadas. Conociendo que el pronóstico y la 
calidad de vida de estos pacientes dependen de sus complicaciones crónicas, es necesario determinar la mejor forma de brindar atención y educación en un Centro Integrado de Salud, que responda a las necesidades de estos pacientes.

Con esta finalidad se creó en 1986 una modalidad de atención denominada "Programa de Enfermedades Crónicas" (PROENCRO) en la Clínica Dr. Marcial Fallas de Desamparados. Además de la consulta médica especializada, se cuenta con una preconsulta, una clínica del pie diabético, un Club de Diabéticos y diversos servicios de apoyo para agilizar el trámite de atención en salud como farmacia y laboratorio.

La Diabetes mellitus tipo 2 se inicia usualmente después de los 40 años y sus complicaciones comienzan a manifestarse casi siempre después de los 55, por lo tanto, es a partir de esta edad que el impacto de la DM, tanto en calidad de vida del paciente como en su necesidad de los servicios de salud en sus tres niveles se viene a acentuar. Se ilustra esto al documentarse que el $56.3 \%$ de los egresos hospitalarios con diagnóstico de DM en 1995 ocurrieron en personas de 60 años y más y el $84.9 \%$ en mayores de 45. ${ }^{2}$ Para conocer el perfil sociodemográfico, de morbilidad, de complicaciones secundarias y de la calidad del control metabólico de los pacientes diabéticos tipo 2 de este grupo etareo, se realizó el presente estudio en una población que recibe atención especializada en una clínica mayor de la Caja Costarricense de Seguro Social. Sus resultados permitirán mejorar la planificación de su atención en salud, tanto en el nivel secundario como en primario, según el modelo actual de la Reforma del Sector Salud, con el fin de mejorar su calidad de vida y que requieran lo mínimo posible ser referidos al nivel terciario.

\section{Materiales y Metódos}

Población y Muestra: PROENCRO inició en 1994 un registro computarizado de todos los pacientes que eran atendidos en la clínica especializada de DM de la Clínica Marcial Fallas en Desamparados. Hasta marzo de 1995 tenía registrado un total de 603 pacientes $(417,186)$, de los cuales $433(71.8 \%)(301,132)$ eran DM tipo 2 con 55 años o más. De estos últimos se tomó una muestra aleatoria de $180(128,52)$ para estudio.

Recolección de Datos: Los datos de evolución de variables clínicas, bioquímicas, de gabinete y la clasificación clínica del pie diabético, se tomaron del expediente clínico del período de abril del 95 a marzo del 96. La clasificación clínica del pie diabético se basó en la clasificación PATONA desarrollada por el Servicio de Medicina Interna del Hospital México. La misma evalua $\boldsymbol{P}$ ulsos, $\boldsymbol{A}$ rcos, $\boldsymbol{T}$ endones, $\boldsymbol{O}$ seo, Neuropatía, $\boldsymbol{A}$ mputación, con puntajes de 0 a 2 cada uno y de acuerdo al puntaje final clasifica los pies como verde, amarillo o rojo.

Los datos socio-demográficos y las medidas antropométricas se completaron en entrevista directa de abril a julio de 1996. El análisis descriptivo se realizó con el programa de SPSS para Windows. La calidad del control metabólico: bueno, regular o malo, se valoró según criterios del manual de tratamiento de la DM tipo 2 de la C.C.S.S. y la Asociación Latinoamericana de Diabetes (ALAD) (Cuadro 1).

\section{Resultados}

Generalidades: Se revisaron los expedientes del total de la muestra, pero se excluyeron 4 individuos del análisis, tres fallecidos antes del período de observación, y un cuarto que no se pudo ubicar. No todas las variables en estudio se encontraban en un $100 \%$, y la n obtenida en cada caso se explica en la descripción específica.

Datos Sociodemográficos: La población diabética estudiada tuvo una relación según sexo de 2.3: 1 predominando las mujeres. La edad media fue de 66 años $\pm 7 .{ }^{6}$ (Cuadro 1 ).

En su mayoría (96.6\%) eran costarricenses por nacimiento, el $63 \%$ de la provincia de San José, pero solo el $34 \%$ oriundo del área atracción en estudio. El estado civil en ambos sexos tuvo un predominio de casados, (hombres $(\mathrm{H})=80 \%$ y mujeres $(\mathrm{M}=$ $42.1 \%$ ), pero también hubo un alto porcentaje de mujeres viudas $(29.2 \%)$.

El $30 \%$ tenían seguro directo, el $45 \%$ familiar y el $28 \%$ por el estado. Este último rubro, es mayor que lo encontrado en el nivel nacional (8.2\%). El grado de instrucción fue bajo con un $87 \%$ con primaria o menos ( $22 \%$ con ninguna escolaridad, $35.2 \%$ con I ciclo y $30 \%$ con II ciclo de primaria). En relación con su condición de actividad, en el sexo femenino fueron mayoritariamente amas de casa $(74 \%)$, con un $24 \%$ pensionadas; en el sexo masculino el $72 \%$ estaba pensionado y el $14 \%$ estaba activo todavía.

Historia Clínica: Los antecedentes heredofamiliares fueron importantes con un $72.2 \%$ para Diabetes (DM) $(M=84 \%$ $\mathrm{F}=67.6 \%$ ) y un $54.5 \%$ para Hipertensión Arterial (HTA).

El rango de edad de inicio de su DM varió de 29 a 78 años, con un promedio de $53.8 \pm 10.1$ años. Un $89 \%$ inició la enfermedad antes de 65 años y un $10 \%$ antes de los 40 años. El promedio de años de evolución fue $12( \pm 8.43)$ con un mínimo de 1 año y un máximo de 31 . Veinte porciento tenía más de 20 años de evolución.

El tratamiento de su DM fue variado (Cuadro 2) con los siguientes resultados:

- $35 \%$ tratamiento único con hipoglicemiantes orales (H.O.), todos con glibenclamida, excepto uno con clorpropamida.

- $62.3 \%$ restante, usaban Insulina NPH, ya sea sola o combinada con Insulina simple o H.O. 


\section{Cuadro 1}

Distribución de la Edad en Quinquenios de los Pacientes DMNID de 55 años y más, según sexo PROENCRO - 1995-96

\begin{tabular}{lcccccc}
\hline \multirow{2}{*}{$\begin{array}{l}\text { Edaden } \\
\text { Quinquenios }\end{array}$} & \multicolumn{2}{c}{ Femenino } & \multicolumn{2}{c}{ Masculino } & \multicolumn{2}{c}{ Total } \\
\cline { 2 - 7 } & $n$ & $\%$ & $n$ & $\%$ & $n$ & $\%$ \\
\hline $55-59$ & 30 & 23.8 & 14 & 28.0 & 44 & 25.0 \\
$60-64$ & 27 & 21.4 & 13 & 26.0 & 40 & 22.7 \\
$65-69$ & 36 & 28.6 & 7 & 14.0 & 43 & 24.4 \\
$70-74$ & 15 & 11.9 & 6 & 12.0 & 21 & 11.9 \\
$75-79$ & 9 & 7.1 & 4 & 8.0 & 13 & 7.4 \\
$80-84$ & 8 & 6.3 & 5 & 10.0 & 13 & 7.4 \\
$85-89$ & 1 & 0.8 & 1 & 2.0 & 2 & 1.1 \\
\hline Total & 126 & 100.0 & 50 & 100.0 & 176 & 100.0 \\
\hline
\end{tabular}

\section{Cuadro 2}

Distribución del Tipo de Tratamiento de los Pacientes DMNID de 55 años o más, PROENCRO, 1995-1996

\begin{tabular}{lcc}
\hline Tratamiento & Frecuencia & Porcentaje \\
\hline Dieta & 3 & 1.7 \\
Glibenclamida 5MG & 15 & 8.6 \\
Glibenclamida 10MG & 17 & 9.7 \\
Glibenclamida 15MG & 14 & 8.0 \\
Glibenclamida 20MG & 14 & 8.0 \\
NPH A.M. & 10 & 5.7 \\
NPHA.M. y P.M. & 40 & 22.9 \\
NPH y H.O. & 26 & 14.9 \\
NPH y simple & 35 & 20 \\
Otro (Clorpropamida) & 1 & .6 \\
\hline Total & 175 & 100.0 \\
\hline
\end{tabular}

- una sola dosis de NPH a.m.: dosis de .26 a .66 unidades por kilo de peso,

- dos dosis NPH a.m. y p.m.: $50 \%$ con dosis totales $>$ a $.6 \mathrm{U} /$ $\mathrm{Kg}$ y $25 \%$ con macrodosis de $.75 \mathrm{U} / \mathrm{kg}$ o más, con dosis máximas de $1.04 \mathrm{U} / \mathrm{Kg}$ de peso.

La comorbilidad fue frecuente en especial con $\operatorname{HTA}(61.9 \%, \mathrm{n}=$ $109)$, obesidad $(41.5 \%, n=73)$ y dislipidemias $(41.5 \%, n=73)$. La cardiopatía isquémica por historia o electrocardiograma fue de un $13 \%(n=23)$, el control psiquiátrico de un $19.9 \%(n=35)$, y el de accidentes vasculares cerebrales con solo 3 casos (2.3\%). El tratamiento para HTA fue variado: el $53.2 \%$ con monoterapia, predominantemente Enalapril ${ }^{R}$ (31\% del total de pacientes

\section{Cuadro 3}

Tipo de medicamento antihipertensivo utilizado tanto en monoterapia como combinada por los 109 pacientes DMNID e hipertensos

\begin{tabular}{lcc}
\hline Tratamiento & Frecuencia & Porcentaje \\
\hline SOLO DIETA & 4 & 3.6 \\
ENALAPRIL 5 MG & 11 & 10.1 \\
ENALAPRIL 20 MG & 30 & 27.5 \\
ATENOLOL 50 MG & 3 & 2.8 \\
ATENOLOL 100 MG & 28 & 25.6 \\
NIFEDIPINA 10 MG & 12 & 11.0 \\
NIFEDIPINA 20 MG & 11 & 10.1 \\
NIFEDIPINA 30 MG & 4 & 3.6 \\
HIDROCLORTIAZIDA25MG & 26 & 23.9 \\
HIDROCLORTIAZIDA5OMG & 16 & 14.7 \\
ALFA METILDOPA & 19 & 17.4 \\
VERAPAMIL & 2 & 1.8 \\
FUROSEMIDE & 7 & 6.4 \\
OTROS & 4 & 3.7 \\
\hline
\end{tabular}

hipertensos); biasociado el $29.4 \%$ (91\% diurético); triasociado el $12 \%$ (Cuadro 3). De los 73 pacientes con dislipidemia, solo 9 personas tenían tratamiento medicamentoso, 7 con Gemfibrozil, uno con Lovastatina y otro con Colestiramina.

Las complicaciones crónicas secundarias a la DM fueron frecuentes. La neuropatía periférica valorada con PATONA, con un $60.8 \%$. La neuropatía autonómica se presentó en 7 pacientes (5.6\%) y todos tenían más de 10 años de evolución, diarrea 6 casos y 1 con taquicardia. Solo el $62 \%$ de los pacientes ( $n=109)$ tenían fondo de ojo actualizado en el periodo de estudio y la prevalencia de retinopatía en éstos fue de un $26.2 \%(n=28)$. Trece casos fueron de tipo proliferativa, 7 de ellos tenían un mínimo de 10 años de evolución y 6 más de 20 años. El $27 \%$ ( $n=$ 48) tenía catarata y en su mayoría eran mayores de 65 años. El $35 \%(n=17)$ había sido operado con una edad promedio de 64 . Los doce casos con catarata que tenían menos de 65 años y tenían más de 15 de evolución de su DM. Seis pacientes informaron ceguera con edades entre 59 y 72; 3 con más de 20 años de evolución, 5 por catarata y 4 , tenían retinopatía diabética.

Solo el $69 \%(n=121)$ tenía valoración de su función renal y la prevalencia de insuficiencia renal crónica fue baja, ( 8 casos, $6.8 \%$ ), igualmente para el síndrome nefrótico con solo dos casos (1.2\%). El mal perforante plantar tuvo una prevalencia del 3.5\% ( 6 casos) y todos tenían un tiempo de evolución mayor de 15 años y un índice de masa corporal $($ IMC) $>30$. Solo dos personas de 61 y 72 años, tenían amputación de miembros inferiores con más de 15 años de evolución.

Perfil Metabólico: En el Cuadro 4 se muestra el número de personas que tenían resultados de cada variable bioquímica en el período estudiado, sus rangos, promedio y desviación

48 AMC, junio 1999, vol 41 (2) 
Cuadro 4

Bioquímica sanguínea de los pacientes DM tipo 2, de 55 y más años

\begin{tabular}{|c|c|c|c|c|c|c|}
\hline Bioquímica Sanguínea & $\mathrm{n}$ & Media & D.E. & E.S. & Mín. & Máx. \\
\hline Glicemia Promedio anual (mg/dl) & 175 & 179.4 & 56.4 & 4.3 & 80.3 & 359.2 \\
\hline Colesterol Total (mg/dl) & 165 & 223.8 & 46.1 & 3.6 & 119 & 402 \\
\hline Trigliceridos (mg/dl) (todos) & 160 & 248 & 171.8 & 13.6 & 65 & 1388 \\
\hline \multicolumn{7}{|l|}{ Triglicéridos (eliminando } \\
\hline "oultiers" $=+3 \cdot d . e)(\mathrm{mg} / \mathrm{dl})$ & 157 & 233.1 & 130.3 & 10.4 & 65 & 768 \\
\hline HDL-Colesterol (mg/dl) & 104 & 42.7 & 9.9 & 1.0 & 25 & 70 \\
\hline LDL- Colesterol (mg/d"' & 104 & 148.4 & 36.0 & 3.5 & 73 & 233 \\
\hline
\end{tabular}

Cuadro 5

Rango Promedio Anual de Gicemia

\begin{tabular}{ccc}
\hline Glicemia & $n$ & $\%$ \\
\hline$<110$ & 11 & 6.3 \\
$111-139$ & 37 & 21.0 \\
$140-199$ & 69 & 39.2 \\
$200-249$ & 35 & 19.9 \\
$250-299$ & 18 & 10.2 \\
$300-349$ & 3 & 1.7 \\
$>350$ & 2 & 1.1 \\
Sin datos & 1 & .6 \\
\hline Total & 176 & 100.0 \\
\hline
\end{tabular}

estándar. Como desglose de la variable principal por intervenir en la DM, se muestra la glicemia promedio anual en rangos (Cuadro 5).

La calidad del control de la diabetes según criterios de ALAD se ilustra en el Cuadro 7.

Perfil Antropométrico: En el cuadro 6 se ilustran las variables antropométricas que muestran el sobrepeso y la distribución de la grasa corporal.

\section{Discusión}

La Diabetes mellitus tipo 2 es actualmente considerada como una patología que se presenta en forma de "epidemia" en la mayoría de los países, y en especial en los del tercer mundo. ${ }^{5}$ Costa Rica no escapa de esta tendencia en el cambio de su perfil epidemiológico en relación con la morbilidad y mortalidad, sobretodo si se considera el envejecimiento rápido de su población. Aunque la población de 60 años y más solo corresponde al $6.7 \%$ del total, genera el $13 \%$ de los egresos hospitalarios, con estancia promedio de 15 días, y la primera causa de egreso corresponde a la DM tipo 2 (un $3.63 \%$ en hombres y un $7.55 \%$ en mujeres). De acuerdo con la consulta externa, la DM ocupa el segundo lugar en este grupo etareo, con un $10 \%$ del total de las consultas, solo superada por la HTA. En un análisis de costo farmacológico de la C.C.S.S. de 1995, en relación con la DM, (Comunicación personal Mora C et al.) el $7.74 \%$ del presupuesto farmacológico del país fue consumido en la atención ambulatoria de los pacientes diabéticos. El gran impacto en costos que tiene esta enfermedad para la seguridad social, y los resultados del perfil del paciente tipo 2 de 55 años descrito en este estudio, muestran una realidad nacional que obliga a reflexionar sobre lo que se le está ofreciendo a los pacientes diabéticos como atención en salud.

Más de dos tercios de todos los pacientes atendidos en PROENCRO tenían 55 años o más en el momento del estudio. De los pacientes estudiados el $89 \%$ había iniciado su DM antes de los 65 años y tenía como promedio 12 años de evolución.

La población de este estudio, se caracterizó por estar predominantemente compuesta por mujeres $(71 \%)$, de baja escolaridad, de ingresos medio y bajo ( $28 \%$ asegurados por el estado), con alta prevalencia de patologías asociadas como hipertensión arterial $(61.9 \%)$, obesidad $(78.5 \%$, incluyendo sobrepeso) y displipidemia (41.5\%). Sus complicaciones secundarias a la DM también fueron altamente prevalentes, con retinopatía en alrededor del $25 \%$ y nefropatía en un $8 \%$. El riesgo de sufrir patología grave (úlceras, mal perforante o amputación) en sus miembros inferiores fue importante, con casi un $45 \%$ de pie amarillo o rojo en PATONA.

El control de su perfil metabólico fue insatisfactorio: el $72.7 \%$ de los pacientes tuvo un promedio anual de glicemias mayor de $140 \mathrm{mg} \%$, nivel relacionado con el desarrollo de la microangiopatía diabética y sus repercusiones; el $75 \%$ tuvo resultados de colesterol total arriba de $200 \mathrm{mg} \%$ y el $67.3 \%$ de LDL-colesterol superior a $130 \mathrm{mg} \%$, cifras consideradas como riesgo para enfermedad coronaria. Su control metabólico en conjunto fue deficiente; en general, todas las variables evaluadas tuvieron un 


\section{Cuadro 6}

Comportamiento de las variables antropométricas

de los pacientes DMNID, de 55 años y más

\begin{tabular}{|c|c|c|c|c|c|c|c|c|}
\hline \multirow{2}{*}{$\begin{array}{l}\text { Variable } \\
\text { Antropométrica }\end{array}$} & \multicolumn{4}{|c|}{ Hombres } & \multicolumn{4}{|c|}{ Mujeres } \\
\hline & Media & D.E. & Mín. & Máx. & Media & D.E. & Mín. & Máx. \\
\hline Peso (Kgs) & 77.9 & 13.6 & 47.1 & 132.5 & 69.1 & 14.6 & 44.0 & 113.2 \\
\hline Talla (cm) & 164.8 & 7.9 & 146.0 & 183.0 & 150.9 & 5.9 & 134.0 & 166.0 \\
\hline Pliegue tricipital (mm) & 9.8 & 4.5 & 4.0 & 28.0 & 15.3 & 5.0 & 6.0 & 30.0 \\
\hline Circunf. cintura $(\mathrm{cm})$ & 100.0 & 9.4 & 81.5 & 140.0 & 92.9 & 10.4 & 74.5 & 123.0 \\
\hline Circunf. cadera $(\mathrm{cm})$ & 105.7 & 9.1 & 85.0 & 134.0 & 109.6 & 11.0 & 89.2 & 141.5 \\
\hline $\begin{array}{l}\text { Indice de Masa Corporal } \\
\left(\mathrm{Kg} / \mathrm{mts}^{2}\right)\end{array}$ & 28.5 & 4.1 & 16.5 & 42.3 & 30.3 & 5.6 & 20.1 & 48.3 \\
\hline Relación cintura/cadera & 0.95 & 0.05 & 0.82 & 1.06 & 0.84 & 0.04 & 0.76 & 0.93 \\
\hline $\begin{array}{l}\text { Circunferencia punto medio } \\
\text { del brazo }(\mathrm{cm})\end{array}$ & 32.9 & 3.8 & 25.0 & 48.5 & 34.3 & 4.5 & 23.3 & 45.0 \\
\hline Circunferencia muscular & & & & & & & & \\
\hline del brazo $(\mathrm{cm})(\mathrm{CMB})$ & 29.5 & 2.9 & 20.9 & 38.3 & 29.6 & 4.0 & 21.4 & 39.6 \\
\hline
\end{tabular}

Cuadro 7

Distribución Porcentual de los Pacientes DMNID de 55 años y más, según Calidad del Control Metabólico, PROENCRO, 1995-1996

\begin{tabular}{lccccc}
\hline & & $\mathrm{n}$ & Bueno & Regular & Malo \\
\hline Glicemia promedio anual & & & $\%$ & $\%$ & $\%$ \\
$\quad$ - En ayunas & $\mathrm{mg} / \mathrm{dl}$ & 175 & 10.9 & 17.7 & 71.4 \\
Colesterol total & $\mathrm{mg} / \mathrm{dl}$ & 165 & 24.2 & 39.9 & 36.4 \\
HDL - Colesterol & $\mathrm{mg} / \mathrm{dl}$ & 104 & 56.7 & 25.0 & 18.3 \\
Triglicéridos en ayunas & $\mathrm{mg} / \mathrm{dl}$ & 171 & 29.8 & 24.0 & 46.2 \\
Indice de masa corporal total & $\mathrm{Kg} / \mathrm{m}^{2}$ & 169 & 16.0 & 14.8 & 69.2 \\
$\quad$ - Hombres & $\mathrm{Kg} / \mathrm{m}^{2}$ & 46 & 19.6 & 17.4 & 63.0 \\
$\quad$ - Mujeres & $\mathrm{Kg} / \mathrm{m}^{2}$ & 123 & 14.5 & 15.1 & 70.4 \\
\hline
\end{tabular}

control malo; solo el HDL-colesterol tuvo un promedio aceptable en sus niveles.

Este panorama, analizado en cuanto a la atención en salud que se ofrece a los pacientes diabéticos, es preocupante, no solo en su control metabólico, sino en la alta prevalencia de enfermedades que son factores de riesgo para la enfermedad cardiovascular, primera causa de muerte en nuestro país. Consideramos que el abordaje de su atención debe enfocarse como un grupo de población de alto riesgo, incluyendo sus familiares, por la alta carga genética encontrada ( $72 \%$ en parientes de primer grado). Además, es necesario, ante nuestra reforma del sector salud y la creación de los Equipo Básico de Atención Integrada en Salud (EBAIS), incorporar en sus actividades, la atención de este grupo poblacional en forma integral, haciendo énfasis en la prevención primaria (cambios de hábitos alimentarios y ejercicio) e intervención secundaria (control adecuado del perfil metabólico y clínico), pero con enfoque de riesgo, en donde intervenga, coordinando y supervisando el nivel II, como parte de un Programa Nacional de Diabetes. Nuevas estrategias deben ser elucidadas, porque lo que hemos hecho hasta hoy, no se muestra halagador.

\section{Abstract}

Diabetes mellitus is a chronic disease whose prevalence will increase with the ageing of the Costa Rican population. The predictive and the quality of life of these patients rely on their chronic complications that usually begin around the sixth decade. Therefore, it is necessary to know the profile of these patient in order to determine the best way to offer them health services that respond to their necessities. In 1986 a Program of Chronic Diseases was created in the outdoor-patient Clinic Dr. Marcial Fallas de Desamparados. Besides the specialized medical consultation, there is a preconsult given by a nurse, a Diabetic Foot clinic and a Diabetic Club. The sociodemographic, pathologic and metabolic control profile of the diabetic patient type 2 of 55 years and over that attends this program is described in this paper.

In 1995 , there was a total of 603 patient registered in the program, of which 432 (71.6\%) were DM type2 patients with 55 years or more. This population was characterized by being more than two thirds women ( $71 \%$ ), of low education, of low economical level ( $28 \%$ in wealth care state policy). There was a high prevalence of chronic pathologies like hypertension $(61.92 \%)$, obesity $(78.5 \%$, including overweight) and

50 AMC, junio 1999, vol 41 (2) 
dyslipidemia (41.5\%). The secondary complications were important: retinopathy in approximately $25 \%$ and nefropathy $8 \%$. The risk of suffering dangerous pathology (ulcers or amputation) in the lower limbs was almost $45 \%$ (yellow Foot in PATONA).

Their metabolic profile was dissapointing: $72 \%$ of the patients had an annual average of glycemias over $140 \mathrm{mg} \%, 75 \%$ had their colesterol > $200 \mathrm{mg} \%$ and $67.3 \%$ had LDL-colesterol> $130 \mathrm{mg} \%$.

Looking at this panorama, we should search for new strategies of attention in health care since this must go further than the common medical attention given up to now in our Clinic. We must put emphassis in health promotion and prevention.

\section{Referencias}

1. Moya L. Estadísticas Generales de los Servicios de Salud. Caja Costarricense de Seguro Social. Serie Estadística de la Salud No 5D. 1996.
2. Sección Información Biomédica C.C.S.S. Egresos por DM en hospitales de la C.C.S.S., Costa Rica, 1995.

3. Depto. Centro de Información. Sección Otros Programas Prioritarios. Min. de Salud, Dirección General de Estadística y Censos. Mortalidad por DM según causa específica y sexo. Costa Rica, 1996.

4. Gries, F.A. Alberti, K.G.M.M. Tratamiento de la Diabetes Mellitus no insulinodependiente en Europa: un informe de consenso. Boletín de la Asociación Latinoamericana de Diabetes, set-dic 1988, 11( 50): 52. (Reproducido al español con permiso del boletín XXXII No. 3 dic 1987 de la Federación Internacional de Diabetes).

5. Pedersen, Terje R, Diabetes y Arteriosclerosis, Número especial del Boletín de la Federación Internacional de Diabetes. vol 42, 1997. 